\title{
Association between miR-27a genetic variants and susceptibility to colorectal cancer
}

\author{
Zaiqiu Wang ${ }^{1,2+}$, Xiaoli Sun ${ }^{3+}$, Yeli Wang ${ }^{2}$, Xiaofang Liư ${ }^{4}$, Yuanjie Xuan ${ }^{2}$ and Sanyuan $\mathrm{Hu}^{1 *}$
}

\begin{abstract}
Background: MicroRNAs (miRNAs) are short, non-coding RNAs that negatively regulate target genes. A single nucleotide polymorphism (SNP) in a miRNA sequence may alter miRNA expression and/or maturation, which was proposed to associate with the development and progression of cancer. The rs895819 polymorphism, located in the terminal loop of pre-miR-27a, has been reported to have relevance to several cancers. In this study, we investigated the possibility of association between polymorphism in rs895819 and susceptibility to colorectal cancer (CRC).
\end{abstract}

Methods: We identified a single SNP, rs895819 in pre-miR-27a, for further investigation, were determined in 205 CRC patients and 455 healthy controls.

Results: When taking the AA genotype as a reference, we found that AG genotype was not statistically significantly associated with the risk of CRC (AG vs. AA, OR 1.245, 95\% Cl: 0.806 - 1.923). However, the GG genotype was significantly associated with risk of CRC (GG vs. AA, OR 1.599, 95\% Cl: 1.052 - 2.430). In the AG + GG vs GG group, no significant difference was detected (OR 1.424, 95\% Cl, $0.974-1.801)$. GG genotype and $\mathrm{G}$ allele was associated with an increased risk of metastasis in this study $(P<0.001$ and $P=0.003$, respectively).

Conclusions: This study found significant association between rs895819 polymorphism in pre-miR-27a and CRC risk. Population-based studies with large number of subjects and long-term follow-up are needed to verify the association of miR-27a polymorphism with CRC susceptibility and severity.

Virtual Slides: The virtual slide(s) for this article can be found here: http://www.diagnosticpathology.diagnomx.eu/vs/ 2061490734125077

Keywords: Single nucleotide polymorphism, Colorectal cancer, miR-27a, Risk factor

\section{Background}

Colorectal cancer $(\mathrm{CRC})$ is reported to be the third most common cancer worldwide with an estimated one million new cases and it is one of the most frequent cancers and a common cause of cancer-related deaths [1-3]. Improvements of diagnosis and treatment had resulted in improved long-term survival rates for patients with early CRC, whereas the outlook for individuals with advanced disease remains poor. Advanced CRC frequently recurs as nodal and hematogenous metastasis and peritoneal dissemination [4]. Although several types of non-surgical treatments have been assessed, surgery is the primary treatment for CRC, and if the tumor is

\footnotetext{
* Correspondence: prhusanyuan@163.com

${ }^{\dagger}$ Equal contributors

'Department of General Surgery, Qilu Hospital of Shandong University, Jinan, Shandong 250012, China

Full list of author information is available at the end of the article
}

caught at a sufficiently early stage, surgery can be curable. The nature of the disease is, however, such that it often presents at an advanced stage, and so surgery alone is insufficient [5].

CRC development involves a multi-step process including both genetic and epigenetic changes, which leads to activation of oncogenes and inactivation of tumor suppressor genes in cancer cells [6]. MicroRNAs (miRNAs) are short, non-coding RNAs of approximately 23 nucleotides that negatively regulate target genes. MiRNAs have been implicated in several biochemical pathways in the eukaryotic cells of various organisms [7]. As a posttranscriptional regulator, miRNAs bind to complementary sequences on target messenger RNAs (mRNAs), resulting in mRNA degradation or translational repression. Sequences encoding the miRNA precursor are usually transcribed by RNA polymerase II to generate primary 
miRNAs (pri-miRNAs). A strong association between miRNA and cancer has been recently reported. MiRNAs have been proposed to contribute to tumorigenesis as either tumor suppressors or oncogenes [8]. A single nucleotide polymorphism (SNP) is a single nucleotide variation of a DNA sequence, $\mathrm{A}$ (adenine), $\mathrm{T}$ (thymine), $\mathrm{C}$ (cytosine), or G (guanine), on genomic DNA. A SNP in a miRNA sequence may alter miRNA expression and/or maturation and be associated with the development and progression of cancer [9].

The relationship between miR-27a and several different cancers have been discussed. Tang et al. reported that the expression of miR-27a was significantly correlated with clinic pathological parameters, including tumor size, lymph node metastasis and distant metastasis, but not with receptor status [10]. Patients with high miR-27a level tended to have significantly shorter disease-free survival durations and overall survival times [10]. It was also reported that the expression of miR-27a was higher in human ovarian cancer relative to benign ovarian tissues. Transfection of SKOV3 cells with the miR-27a inhibitor could suppress the growth and migration of tumor cells [11].

We utilized public databases to identify deregulated miRNAs in CRC and SNPs in these miRNAs sequences, including primary, precursor and mature miRNAs. Our searches identified a single SNP, rs895819 in pre-miR-27a, for further investigation. The rs895819 polymorphism is located in the terminal loop of pre-miR-27a and involves an AG nucleotide transition. The association between rs895819 polymorphism and risk of several cancers were reported [12-14]. In this study, we investigated the possibility of association between polymorphism in rs895819 and susceptibility to CRC.

\section{Methods}

\section{Ethics statement}

This study was approved by the institutional review board of Qilu Hospital of Shandong University. All the participants were voluntary and provided written informed consent prior to taking part in this research.

\section{Study subjects}

All subjects were recruited from Qilu Hospital of Shandong University between January 2010 and December 2013. They were all newly diagnosed, histopathologically confirmed and without a prior history of cancer or previous chemo- or radiotherapy. In all, 205 patients with CRC were recruited, all of whom were unrelated ethnic Han Chinese population. A structured questionnaire on demographics and environmental exposure, including age, sex, smoking consumption and alcohol drinking, was conducted by trained interviewers through face-to-face interviews with the patients. In addition, $5 \mathrm{ml}$ venous blood was collected from each patient for genomic DNA extraction. The clinical characters of all cases were recorded by reviewing the databases or counseling the doctor.

\section{DNA extraction}

For both the cases and control cohorts, $1.5 \mathrm{ml}$ of whole blood was extracted from each participant and stored at $-80^{\circ} \mathrm{C}$ before being used. DNA from each sample of whole blood was extracted with the QIAamp DNA mini Kit (Qiagen, Hilden, Germany), as directed by the manufacturer's instructions. The concentration of DNA and the purity of each sample were measured by an ultraviolet spectrophotometer (GE Healthcare, USA). DNA samples were routinely stored at $-20^{\circ} \mathrm{C}$.

\section{SNP genotyping analysis}

Genotyping was performed by TaqMan allelic discrimination assays using an ABI 7900 system (Applied Biosystems, Foster City, CA, USA). The prime and probes are as follows: forward, GGCGGAACTTAGCCACTGT, reverse, CAGGGCTTAGCTGCTTGTG. The TaqMan assays were performed in a final reaction volume of $5 \mathrm{ml}$ containing $0.25 \mathrm{ml}$ primer, $0.125 \mathrm{ml}$ probe, $2 \mathrm{ml}$ PCR mixture reagent and 25 ng DNA. The PCR reaction consisted of an initial step at $95 \mathrm{uC}$ for $10 \mathrm{~min}$ followed by 55 cycles of denaturing at $95 \mathrm{uC}$ for $15 \mathrm{~s}$ and annealing at $60 \mathrm{uC}$ for $60 \mathrm{~s}$. SDS allelic discrimination software (ABI) was used to analyze the PCR genotyping results. Two blank (water) controls were included in each 384-well assay. At least 10\% of samples were randomly selected for repeat analysis, yielding 100\% concordance. A further 60 samples were selected randomly for direct sequencing to confirm the TaqMan results. Again, the results showed $100 \%$ concordance.

\section{Statistical analysis}

Statistical analysis was performed using the SPSS 20.0 software package (SPSS Company, Chicago, IL). Hardy-Weinberg equilibrium analysis was performed using a goodness-of-fit $X^{2}$-test with one degree of freedom. $\mathrm{P}<0.05$ was considered representative of a departure from HWE. Comparison of allele type and genotype distributions in the patients and healthy controls were performed by means of two-sided contingency tables using the $X^{2}$-test. The odds ratio (OR) and 95\% confidence interval (CI) for genotypic-specific risk were calculated using an unconditional logistic regression model. $\mathrm{P}<0.05$ was considered significant for all statistical analyses.

\section{Result}

General characteristics of the subjects

A total of 205 CRC patients and 455 healthy controls were enrolled in our study. All of the subjects were ethnic Han Chinese. Demographic and other selected 
Table 1 General characteristics of colorectal cancer (CRC) patients and controls

\begin{tabular}{|c|c|c|c|c|c|c|}
\hline Variable s & Cases & Percentage (\%) & Control & Pecentage (\%) & $P$ value & OR $(95 \% \mathrm{Cl})$ \\
\hline Age (years, mean $\pm S D$ ) & $54.9 \pm 11.2$ & & $55.4 \pm 10.1$ & & 0.15 & \\
\hline \multicolumn{7}{|l|}{ Sex } \\
\hline Male & 103 & 50.24 & 225 & 49.45 & & 1 (Ref) \\
\hline Female & 102 & 49.76 & 230 & 50.55 & 0.859 & $1.032(0.742-1.435)$ \\
\hline \multicolumn{7}{|l|}{ Smoking status } \\
\hline No & 61 & 29.76 & 201 & 44.18 & & 1 (Ref) \\
\hline Yes & 144 & 70.24 & 254 & 55.82 & $<0.001$ & $1.868(1.314-2.656)$ \\
\hline \multicolumn{7}{|c|}{ Smoking amount (pack-years) } \\
\hline 0 & 61 & 29.76 & 201 & 44.18 & & 11 (Ref) \\
\hline$<1500$ & 51 & 24.87 & 123 & 27.03 & 0.453 & $1.21(0.88-2.11)$ \\
\hline$>1500$ & 93 & 45.36 & 131 & 28.79 & $<0.001$ & $2.34(1.58-3.46)$ \\
\hline \multicolumn{7}{|l|}{ Smoking duration (years) } \\
\hline 0 & 61 & 29.76 & 201 & 44.18 & & (Ref) 1 \\
\hline$<5$ & 12 & 5.85 & 37 & 8.13 & 0.325 & $1.069(0.525-2.177)$ \\
\hline $5-10$ & 39 & 19.02 & 81 & 17.80 & 0.067 & $1.587(0.984-2.558)$ \\
\hline$>10$ & 93 & 45.36 & 150 & 32.97 & 0.002 & $2.43(1.389-3.005)$ \\
\hline \multicolumn{7}{|l|}{ Alcohol } \\
\hline No & 89 & 43.41 & 236 & 51.87 & & 1 (Ref) \\
\hline Yes & 116 & 56.59 & 219 & 48.13 & 0.044 & $1.405(1.008-1.957)$ \\
\hline \multicolumn{7}{|c|}{ Alcohol amount (cups/day) } \\
\hline 0 & 89 & 43.41 & 236 & 51.87 & & 1 (Ref) \\
\hline $1-3$ & 39 & 19.02 & 102 & 22.42 & 0.284 & $1.014(0.651-1.578)-$ \\
\hline$>3$ & 77 & 38.54 & 117 & 26.71 & 0.012 & $1.790(1.230-2.606)$ \\
\hline \multicolumn{7}{|l|}{ Alcohol use duration } \\
\hline 0 & 89 & 43.41 & 236 & 51.87 & & 1 (Ref) \\
\hline$<5$ & 32 & 15.61 & 78 & 17.53 & 0.329 & $1.088(0.674-1.755)$ \\
\hline $5-10$ & 29 & 14.15 & 65 & 14.61 & 0.436 & $1.183(0.717-1.953)$ \\
\hline$>10$ & 55 & 26.83 & 76 & 17.08 & $<0.001$ & $1.919(1.256-2.933)$ \\
\hline \multicolumn{7}{|l|}{ Fruit intake (times/week) } \\
\hline$\leq 1$ & 68 & 33.17 & 120 & 26.97 & & 1 (Ref) \\
\hline $2-4$ & 53 & 25.85 & 165 & 37.07 & 0.042 & $0.588(0.380-0.899)$ \\
\hline$\geq 5$ & 84 & 40.98 & 170 & 38.20 & 0.245 & $0.872(0.587-1.296)$ \\
\hline \multicolumn{7}{|c|}{ Vegetable intake (times/week) } \\
\hline$\leq 1$ & 85 & 41.46 & 116 & 26.07 & & 1 (Ref) \\
\hline $2-4$ & 75 & 36.59 & 131 & 29.43 & 0.546 & $0.781(0.524-1.164)$ \\
\hline$\geq 5$ & 45 & 21.95 & 198 & 44.49 & $<0.001$ & $0.310(0.202-0.476)$ \\
\hline \multicolumn{7}{|l|}{ Education status } \\
\hline High school or less & 109 & & 228 & & & 1 (Ref) \\
\hline College or University & 81 & & 202 & & & $0.839(0.594-1.183)$ \\
\hline Master or more & 15 & & 15 & & & $2.092(0.987-4.434)$ \\
\hline \multicolumn{7}{|l|}{ Income (RMB/year) } \\
\hline$\leq 100,000$ & 98 & 47.80 & 109 & 24.49 & & 1 (Ref) \\
\hline $100,000-300,000$ & 88 & 42.93 & 252 & 56.12 & 0.001 & $0.388(0.270-0.560)$ \\
\hline$\geq 300,000$ & 19 & 9.27 & 84 & 18.88 & $<0.001$ & $0.252(0.143-0.444)$ \\
\hline
\end{tabular}


characteristics of cases and controls were presented in Table 1. Cases and controls did not show statistically significant differences with regard to sex and age. However, the higher smoking and alcohol drinking were risks of CRC $(\mathrm{P}=0.001, \quad \mathrm{OR}=1.868 ; \quad \mathrm{P}=0.044, \quad \mathrm{OR}=1.405$, respectively). When the duration or amount was considered, higher amount (OR, 2.34, 95\% CI, 1.58 -3.46) and duration (OR, 2.43; 95\% CI, $1.389-3.005)$ of tobacco smoking were associated with the risk of CRC. Besides, the higher amount of alcohol consumption was associated with higher risk of CRC (OR, 1.790; 95\% CI, 1.230 - 2.606). In the advanced analyses, the results showed that high intake of vegetable (OR, 0.310 ; $95 \% \mathrm{CI}, 0.202-0.476)$ and fruit intake (OR, 0.588; 95\% CI, $0.380-0.899$ ) was associated with reduced risk of CRC incidence. The education statuses were not associated with the risk of $\mathrm{CRC}$, while lower income might lead to high incidence of CRC (OR, 0.252; 95\% CI, 0.143 - 0.444).

With regard to clinic pathologic characteristics of CRC patients, most patients had a smaller tumor size (less than $5 \mathrm{~cm}, 65.85 \%$ ). Well-moderately status was in $126(61.46 \%)$ patients, while poorly-undifferentiated status was in 79 (38.54\%) patients. A total of 86 patients (41.95\%) had metastasis among all the patients. According to the TNM classification criteria, 138 and 67 patients were in stage I-II and III-IV, respectively (Table 2).

Table 2 Clinical characteristics of colorectal cancer (CRC) patients and controls

\begin{tabular}{lcc}
\hline Variables & Cases & Percentage (\%) \\
\hline Tumor size & 135 & 65.85 \\
$<5 \mathrm{~cm}$ & 70 & 34.15 \\
$\geq 5 \mathrm{~cm}$ & & \\
Differentiated status & 126 & 61.46 \\
Well-moderately & 79 & 38.54 \\
Poorly-Undifferentiated & & \\
TNM stage & 138 & 67.32 \\
I-II & 67 & 32.68 \\
III- IV & & \\
Metastasis & 86 & 41.95 \\
Yes & 119 & 58.05 \\
No & & \\
Site & 108 & 52.68 \\
Colon & 73 & 35.61 \\
Rectum & 24 & 11.71 \\
Both & & \\
Therapy & 126 & 61.46 \\
Sugery & 23 & 11.21 \\
Local resection & 56.31 \\
Chemotherapy & & \\
\hline
\end{tabular}

\section{The effect of the rs895819 polymorphism on the risk} of CRC

The genotype and allele frequencies of the rs895819 were presented in Table 3. The genotype frequencies in both cases and controls were in agreement with the HWE model. As shown in Table 3, the genotype frequencies of the rs895819 were $11.71,39.02$ and $49.27 \%$ for the AA, AG, and GG genotypes respectively, among the CRC group; and 30.33, 34.51 and $35.16 \%$ among the controls, respectively. When taking the AA genotype as a reference, we found that AG genotype was not statistically significantly associated with the risk of CRC (AG vs. AA, OR 1.245, 95\% CI: 0.806 - 1.923). However, compared with AA genotype, the GG genotype was significantly associated with risk of CRC (GG vs. AA, OR 1.599, $95 \% \mathrm{CI}: 1.052-2.430)$. In the $\mathrm{AG}+\mathrm{GG}$ vs GG group, no significant difference was detected (OR 1.424, 95\% CI, 0.974 - 1.801).

Besides, the allele distribution was significant difference between cases and controls (OR 1.362, 95\% CI 1.075 1.725). Taken together, these data suggest that the miR-27a binding site polymorphism rs895819 may be associated with the risk of CRC.

\section{The rs895819 polymorphism and clinicopathological characteristics}

In the stratified studies, we attended to detect the association between rs895819 polymorphism and clinicopathological characteristics of patients with CRC. We observed no association between rs895819 polymorphism and the progression of CRC including tumor size and tumor stage. However, GG genotype and G allele was associated with an increased risk of metastasis in this study $(\mathrm{P}<0.001$ and $\mathrm{P}=0.003$, respectively) (Table 4 ).

\section{Discussion}

According to the polygenic model of carcinogenesis, unfavorable combinations of polymorphic genetic variants in low-penetrance susceptibility genes contribute to increasing CRC risk. Studies have shown that profiles of miRNA expression differ between normal and tumor tissues, which vary among tumor types and the down-regulation of miRNA subsets implies a tumorsuppressor function, which is often observed in tumor development. Recently, a class of novel functional polymorphisms in miRNAs or their binding sites has attracted great interest. One important SNP in pre-mir-27a of an A to $G$ change was identified as rs895819, and its role in cancer susceptibility has been discussed recently $[14,15]$. Though many studies have been performed, whether rs895819 contributes to cancer susceptibility is still unclear, and the association between rs895819 and CRC risk remains unknown. Therefore, our present study investigated the association of rs895819 with 
Table 3 Genotypes of rs895819 polymorphism and CRC risk

\begin{tabular}{|c|c|c|c|c|c|c|}
\hline Genotype & Cases & Percentage (\%) & Control & Pecentage (\%) & $P$ value & OR $(95 \% \mathrm{Cl})$ \\
\hline$\overline{A A}$ & 48 & 11.71 & 138 & 30.33 & & 1 (Ref) \\
\hline AG & 68 & 39.02 & 157 & 34.51 & 0.087 & $1.245(0.806-1.923)$ \\
\hline GG & 89 & 49.27 & 160 & 35.16 & 0.015 & $1.599(1.052-2.430)$ \\
\hline$A G+G G$ & 157 & 88.29 & 317 & 69.67 & 0.064 & $1.424(0.974-1.801)$ \\
\hline \multicolumn{7}{|c|}{ Allele frequency } \\
\hline A allele & 164 & 32.22 & 433 & 49.58 & & 1 (Ref) \\
\hline G allele & 246 & 67.78 & 477 & 50.42 & 0.027 & $1.362(1.075-1.725)$ \\
\hline
\end{tabular}

CRC risk in the Chinese population. In a case-control study with 205 cases and 455 controls, our study demonstrated that subjects with GG genotype and the $G$ allele exhibited a significantly increased risk of CRC in the cohort. Advanced study on the association between rs895819 and the clinical pathological characters showed that GG genotype and the $G$ allele were risk factors of increased risk of CRC.

A previous study by $\mathrm{Fu}$ et al. identified 32 differentially expressed miRNAs and 2916 mRNAs from CRC samples and their corresponding normal epithelial tissues by miRNA and mRNA microarray, respectively. Additionally, 6 up-regulated miRNAs (mir-21, mir-223, mir-224, mir-29a, mir-29b, and mir-27a) and 4 down-regulated predicted target mRNAs (SFRP1, SFRP2, RNF138, and KLF4) were selected to validate the expression level and their anti-correlationship in an extended cohort of CRC patients by qRT-PCR [16]. In animal model, it was also reported that a recurrent amplicon on mouse chromosome 8 that encodesmiR-23a and -27a levels are up-regulated in mouse intestinal adenocarcinomas, primary tumors from patients with stage I/II colorectal cancers, as well as in human colorectal cancer cell lines and cancer stem cells [11]. It could be presumed that miR-27a might play a key role in the development and progression of CRC. Some more studies were also

Table 4 Clinicopathological characteristics and miR-27a genotype and allelic frequencies of CRC patients

\begin{tabular}{|c|c|c|c|c|c|c|c|c|}
\hline & \multicolumn{3}{|c|}{ Genotype } & \multicolumn{2}{|c|}{$P$ value } & \multicolumn{2}{|c|}{ Allele } & \multirow[b]{2}{*}{$P$ value } \\
\hline & $A A$ & AG & GG & AG vs $A A$ & GG vs $A A$ & A & G & \\
\hline \multicolumn{9}{|c|}{ Tumor size } \\
\hline$<5 \mathrm{~cm}$ & 33 & 40 & 62 & & & 103 & 162 & \\
\hline$\geq 5 \mathrm{~cm}$ & 16 & 20 & 34 & 0.784 & 0.812 & 52 & 88 & 0.734 \\
\hline \multicolumn{9}{|c|}{ TNM stage } \\
\hline$|-| \mid$ & 32 & 43 & 60 & & & 105 & 163 & \\
\hline III- IV & 15 & 21 & 34 & 0.729 & 0.617 & 51 & 89 & 0.587 \\
\hline \multicolumn{9}{|c|}{ Metastasis } \\
\hline Yes & 19 & 20 & 47 & & & 58 & 114 & \\
\hline No & 28 & 31 & 69 & 0.903 & $<0.001$ & 87 & 169 & 0.003 \\
\hline
\end{tabular}

conducted to identify the potential role of miR-27a in CRC. Kim et al. showed that human miR-27a* is a negative regulator of NK-cell cytotoxicity by silencing Prf1 and GzmB expression. Human miR-27a* specifically bound to the 3' untranslated regions of Prf1 and GzmB, down-regulating expression in both resting and activated NK cells, and it functioned as a fine-tuner for homeostasis of the net amount of the effectors proteins. Consistent with miR-27a* having an inhibitory role, knockdown of miR-27a* in NK cells dramatically increased cytotoxicity in vitro and decreased tumor growth in a human tumor xenograft model. Thus, NK-cell cytotoxicity is regulated, in part, by microRNA, and modulating endogenous miR-27a* levels in NK cells represents a potential immunotherapeutic strategy [17]. Resveratrol and quercetin (RQ) in combination (1:1 ratio) could produce a significant anticancer effect through decreasing the miR-27a level in colon cancer cell lines [18]. This was confirmed by transfection of cells with the specific mimic for miR-27a, which partially reversed the effects of $R Q$.

Single nucleotide polymorphism of miRNA would influence the expression of miRNA and thus would influence the cancer incidence or progression. Zhou et al. [19] reported that 19 SNPs of the miRNA-related genes in a case-control study of 311 gastric cancers and 425 cancer-free controls in a Chinese Han population. The results showed that the SNP rs895819 in the miR-27a gene with the minor allele $C$ presented significantly reduced risk to gastric cancer $(\mathrm{p}=0.037, \mathrm{OR}=0.771$, $95 \% \mathrm{CI}=0.604-0.985)$. Wang et al. [20] conducted a meta-analysis of miR-27a SNP and risk of breast cancer. They found that hsa-mir-27a rs895819 polymorphism also did not reveal any relationship with breast cancer susceptibility, while significantly decreased risk was found among Europeans in AG versus $\mathrm{AA}$ and $\mathrm{AG} / \mathrm{GG}$ versus $\mathrm{AA}$ models tested (AG versus AA: $\mathrm{OR}=0.83$; 95\% CI, 0.72-0.97; GG versus $\mathrm{AA}$ : $\mathrm{OR}=0.86 ; 95 \% \mathrm{CI}, 0.71-1.05 ; \mathrm{AG} / \mathrm{GG}$ versus $\mathrm{AA}: \mathrm{OR}=0.84 ; 95 \% \mathrm{CI}, 0.75-0.94$ ).

The association between miR-27a SNP and CRC risk was also studied in several studies. Hezova $\mathrm{R}$ et al. [21] conducted a case-control study to investigate whether selected single nucleotide polymorphisms (SNPs) in 
miR-196a2, miR-27a and miR-146a genes were associated with sporadic colorectal cancer (CRC). The results of this case-control study of 197 cases of sporadic CRC and 212 cancer-free controls originating from the Central-European Caucasian population showed that there was a lack of association between rs11614913, rs895819 and rs2910164 and colorectal cancer risk in the Central-European Caucasian population. This result was contradictory with our study. The potential explanations were the difference between the locality and races included in the two studies. The genetic background and environmental factors might influence the effect of rs895819 on the risk of CRC.

\section{Conclusion}

Our results showed an association between miR-27a polymorphism and CRC in Chinese Han patients. Besides, miR-27a polymorphism showed a significant association with the risk of metastasis. However, no functional validation of these observational findings was conducted and a series of following studies would be conducted. Considering that this is from a case-control study, however, populationbased studies with large number of subjects and long-term follow-up are needed to verify the association of miR-27a polymorphism with CRC susceptibility and severity.

\section{Competing interests}

The authors declare that they have no competing interests.

\section{Authors' contribution}

WZQ, WYL and HSY provided the conduction of the whole project, WZQ, $S X L, W Y L, L X F, X Y$ J and HSY performed the research, WZQ, SXL, WYL, LXF, XYJ and HSY drafted the manuscript; WZQ, SXL, WYL, LXF, XYJ and HSY contributed to revise the manuscript. All authors read and approved the final manuscript.

\section{Authors' information}

Zaiqiu Wang and Xiaoli Sun are co-first authors.

\section{Acknowledgement}

No acknowledgements should be stated.

\section{Author details}

'Department of General Surgery, Qilu Hospital of Shandong University, Jinan, Shandong 250012, China. 'Department of Anorectal, Yuhuangding Hospital, Yantai, Shandong 264000, China. ${ }^{3}$ Department of Laboratory, Yuhuangding Hospital, Yantai, Shandong 264000, China. ${ }^{4}$ Department of Hepatobiliary, Yuhuangding Hospital, Yantai, Shandong 264000, China.

Received: 25 March 2014 Accepted: 13 June 2014

Published: 30 July 2014

\section{References}

1. Fan NJ, Kang R, Ge XY, Li M, Liu Y, Chen HM, Gao CF: Identification alpha-2-HS-glycoprotein precursor and tubulin beta chain as serology diagnosis biomarker of colorectal cancer. Diagn Pathol 2014, 9:53.

2. Chen C, Wang L, Liao Q, Huang Y, Ye H, Chen F, Xu L, Ye M, Duan S: Hypermethylation of EDNRB promoter contributes to the risk of colorectal cancer. Diagn Pathol 2013, 8:199.

3. Schmoll HJ, Stein A: Colorectal cancer in 2013: Towards improved drugs, combinations and patient selection. Nat Rev Clin Oncol 2014, 11:79-80.

4. Yatabe J, Yatabe MS, Ishibashi K, Nozawa Y, Sanada H: Early detection of colon cancer by amino acid profiling using Aminolndex Technology: a case report. Diagn Pathol 2013, 8:203.
5. Wang L, Cai S, Teng Z, Zhao X, Chen X, Bai X: Insulin therapy contributes to the increased risk of colorectal cancer in diabetes patients: a meta-analysis. Diagn Pathol 2013, 8:180.

6. Liu F, Xiong Y, Zhao Y, Tao L, Zhang Z, Zhang H, Liu Y, Feng G, Li B, He L, Ma J, Qin S, Yang Y: Identification of aberrant microRNA expression pattern in pediatric gliomas by microarray. Diagn Pathol 2013, 8:158.

7. Wang S, Li H, Wang J, Wang D: Expression of microRNA-497 and its prognostic significance in human breast cancer. Diagn Pathol 2013, 8:172.

8. Qin W, Zhang K, Clarke K, Weiland T, Sauter ER: Methylation and miRNA Effects of Resveratrol on Mammary Tumors vs Normal Tissue. Nutr Cancer 2014, 66:270-277.

9. Zhou J, Yu L, Gao X, Hu J, Wang J, Dai Z, Wang JF, Zhang Z, Lu S, Huang X, Wang Z, Qiu S, Wang X, Yang G, Sun H, Tang Z, Wu Y, Zhu H, Fan J: Plasma microRNA panel to diagnose hepatitis $B$ virus-related hepatocellular carcinoma. J Clin Oncol 2011, 29:4781-4788.

10. Tang W, Zhu J, Su S, Wu W, Liu Q, Su F, Yu F: MiR-27 as a prognostic marker for breast cancer progression and patient survival. PLOS One 2012, 7:e51702.

11. Jahid S, Sun J, Edwards RA, Dizon D, Panarelli NC, Milsom JW, Sikandar SS, Gümüs ZH, Lipkin SM: miR-23a promotes the transition from indolent to invasive colorectal cancer. Cancer Discov 2012, 2:540-553.

12. Kupcinskas J, Wex T, Link A, Leja M, Bruzaite I, Steponaitiene R, Juzenas S, Gyvyte U, Ivanauskas A, Ancans G, Petrenkiene V, Skieceviciene J, Kupcinskas L, Malfertheiner P: Gene polymorphisms of micrornas in Helicobacter pylori-induced high risk atrophic gastritis and gastric cancer. PLoS One 2014, 9:e87467.

13. Catucci I, Verderio P, Pizzamiglio S, Bernard L, Dall'olio V, Sardella D, Ravagnani F, Galastri L, Barile M, Peissel B, Zaffaroni D, Manoukian S, Radice P, Peterlongo P: The SNP rs895819 in miR-27a is not associated with familial breast cancer risk in Italians. Breast Cancer Res Treat 2012, 133:805-807.

14. Yang R, Schlehe B, Hemminki K, Sutter C, Bugert P, Wappenschmidt B, Volkmann J, Varon R, Weber BH, Niederacher D, Arnold N, Meindl A, Bartram CR, Schmutzler RK, Burwinkel B: A genetic variant in the pre-miR-27a oncogene is associated with a reduced familial breast cancer risk. Breast Cancer Res Treat 2010, 121:693-702.

15. Sun Q, Gu H, Zeng Y, Xia Y, Wang Y, Jing Y, Yang L, Wang B: Hsa-mir-27a genetic variant contributes to gastric cancer susceptibility through affecting miR-27a and target gene expression. Cancer Sci 2010, 101:2241-2247.

16. Fu J, Tang W, Du P, Wang G, Chen W, Li J, Zhu Y, Gao J, Cui L: Identifying microRNA-mRNA regulatory network in colorectal cancer by a combination of expression profile and bioinformatics analysis. BMC Syst Biol 2012, 6:68

17. Kim TD, Lee SU, Yun S, Sun HN, Lee SH, Kim JW, Kim HM, Park SK, Lee CW, Yoon SR, Greenberg PD, Choi I: Human microRNA-27a* targets Prf1 and GzmB expression to regulate NK-cell cytotoxicity. Blood 2011, 118:5476-5486.

18. Del Follo-Martinez A, Banerjee N, Li X, Safe S, Mertens-Talcott S: Resveratrol and quercetin in combination have anticancer activity in colon cancer cells and repress oncogenic microRNA-27a. Nutr Cancer 2013, 65:494-504.

19. Zhou Y, Du WD, Chen G, Ruan J, Xu S, Zhou FS, Zuo XB, Lv ZJ, Zhang XJ: Association analysis of genetic variants in microRNA networks and gastric cancer risk in a Chinese Han population. J Cancer Res Clin Oncol 2012, 138:939-945

20. Wang $B$, Ma N, Wang Y: Association between the hsa-mir-27a variant and breast cancer risk: a meta-analysis. Asian Pac J Cancer Prev 2012, 13:6207-6210.

21. Hezova R, Kovarikova A, Bienertova-Vasku J, Sachlova M, Redova M, Vasku A, Svoboda M, Radova L, Kiss I, Vyzula R, Slaby O: Evaluation of SNPs in miR-196-a2, miR-27a and miR-146a as risk factors of colorectal cancer. World J Gastroenterol 2012, 18:2827-2831.

doi:10.1186/1746-1596-9-146

Cite this article as: Wang et al:: Association between miR-27a genetic variants and susceptibility to colorectal cancer. Diagnostic Pathology 2014 9:146. 